\title{
FLORA RUDERAL DA CIDADE DE CUIABÁ, MATO GROSSO, BRASIL
}

\author{
Débora Eriléia Pedrotti' \\ Germano Guarim Neto ${ }^{2}$
}

Recebido em 21/05/96. Aceito em 22/05/98

\begin{abstract}
RESUMO - (Flora ruderal da cidade de Cuiabá, Mato Grosso. Brasil). O estudo sobre a flora ruderal de cidades. atualmente, tem sido intensificado, tendo como objetivo primordial o conhecimento do seu potencial e as variações na sua utilização. A literatura, até o momento, não registrava nenhum trabalho sobre este aspecto. para a cidade de Cuiabá (Mato Grosso). Tendo em vista a alta ocorrência de espécies ruderais nesta cidade, realizou-se o presente estudo com o objetivo de catalogar a sua flora ruderal. bem como verificar os usos regionais das espécies. As coletas foram realizadas em duas áreas: Campus da Universidade Federal de Mato Grosso e o bairro Boa Esperança. A triagem dos vegetais. bem como as identificações, foram realizadas no Laboratório de Botânica do Instituto de Biociências. desta Universidade. O levantamento botânico eletuado nas duas áreas catalogou 109 espécies e 81 gêneros, distribuídos por 35 famílias. A família Gramineae (=Poaceae) apresentou o maior número de espécies (14), seguida de Compositac (=Asteraceac 13), Leguminosac (=Fabaceae 11), Euphorbiaceae (10) e outras com menor número. Entre as 109 espécies catalogadas pode-se citar Panicum maximum Jacq. (capim-colonião). Turnera ulmifolia L. (chanana). Cecropia peltata L. (embaúva), Ipomoea acuminata Roem \& Sch. (campainha). as quais são utilizadas localmente como plantas forrageiras, ornamentais e medicinais.
\end{abstract}

Palavras-chave - plantas ruderais, Cuiabá, Mato Grosso, Ievantamento florístico

ABSTRACT - (Ruderal plants from Cuiabá city. Mato Grosso. Brazil). Studies about ruderal plants in cities have actually been intense, having as a primordial objective the knowledge of its potential and variations in its utilization. Any work about this aspect in the city of Cuiabá (Mato Grosso - Brazil) hasn't been found in the literature so far. Owing to the high occurrence of ruderal species in this city, this study was realized aiming at cataloguing them. as well as verifying their regional use. The sampling was realized in two selected areas, the "Universidade Federal de Mato Grosso" Campus and the "Boa Esperança" neighbourhood. The plants selection and their identification were realized in the Botany Lahoratory of the Biosciences Institute of this University. The botanical survey undertaken in these two areas catalogued 109 species and 81 genera distributed in 35 families. The family Gramineae (=Poaceae) presented the highest number of species (14), followed by Compositae (=Asteraceae 13), Leguminosae (=Fabaceae 11). Euphorbiaceac (10) and others with lower number. Among the 109 catalogued species,

Bióloga (ex Bolsista IC/CNPq)

Departamento de Botânica e Ecologia. Instituto de Biociências. Universidade Federal de Mato Grosso, CEP 78060-900. Cuiabá, MT. Bolsista CNPq. e-mail: guarim@cpd.ufmt.br 
Panicum maximum Jacq. (capim-colonião), Turnera ulmifolia L. (chanana), Cecropia peltata

L. (embaúva) and Ipomoea acuminata Roem \& Sch. are used locally as fodder, adornment and in popular medicine.

Key words - ruderal plants. Mato Grosso, floristic survey

\section{Introdução}

Segundo Rizzini (1979), ruderal provém de rudera, plural: ruínas e qualifica os vegetais que vivem em áreas ligadas aos habitats humanos, como terrenos baldios ou devastados, caminhos, estradas, ruas, jardins. Têm sido designados como nitrófilos, porque suas estações, próximas ao homem dispõem de maiores quotas de nitrogênio. Tal designação, porem, tem preferentemente sentido geral, nomeando qualquer planta que exija concentração definida de nitrogênio, relativamente elevada. Tendo em vista estes aspectos, a flora ruderal de cidades vem sendo tratada em diferentes estudos no país, como demonstram Amaral et al. (1957), no Rio Grande do Sul; Carvalho (1958), no Rio de Janeiro; Correia \& Chaves (1972), em João Pessoa, Paraíba; Gonçalves et al. (1974), no Pará; Ferreira \& Laca-Buendia (1978), em Minas Gerais; Albuquerque (1980), na região de Manaus, Amazonas; Mautone et al. (1990). no Rio de Janeiro; Gavilanes \& D’Angieri Filho (1991), na cidade de Lavras, Minas Gerais.

A flora ruderal está constituída de espécies adaptadas a ambientes alterados, como margens de estradas, proximidades de habitações abandonadas ou não, loteamentos urbanos, terrenos baldios e similares. Leitão Filho et al. (1972) e Lorenzi (1991) definem como plantas ruderais os componentes da vegetação urbana que crescem espontaneamente na margem de ruas, sobre muros, telhados e calçadas e que quase sempre se comportam como indesejáveis. As comunidades ruderais estão sob condições distintas daquelas que compõem ecossistemas naturais, incluindo diferenças nas próprias populações e nos ambientes físicos e bióticos que elas ocupam. A mais importante diferença entre esses ecossistemas está na intensidade da interferência humana (Snaydon 1962).

De acordo com Grime (1982) e Lorenzi (1991), a extrema habilidade das plantas ruderais quanto à sobrevivência é atribuída a atributos ou mecanismos desenvolvidos pela natureza: grande agressividade competitiva, grande produção e longevidade das sementes.

Segundo Lorenzi (1984), as plantas daninhas, quando crescem juntamente com outras culturas, interferem no seu desenvolvimento, reduzindo-lhes a produção. Competem pela extração dos elementos vitais como água, luz, $\mathrm{CO}_{2}$ e nutrientes, exercendo inibição química sobre o desenvolvimento das plantas. Podem ainda comprometer indiretamente culturas agrícolas por hospedarem pragas e doenças antes de infestarem as próprias lavouras. Na pecuária, as plantas daninhas interferem com as forrageiras reduzindo a capacidade de lotação das pastagens. Algumas causam ferimentos mecânicos nos animais e outras conferem gosto desagradável ao leite, se ingeridas por vacas em lactação. Por outro lado, também causam transtornos na saúde e na própria vida do homem. Em beira de estradas e rodovias obstruem a visibilidade e são reponsáveis por incêndios freqüentes, não raro provocando, em consequiência, sérios acidentes. Em 
logradouros públicos impedem a recreação infantil e em terrenos baldios, via de regra, servem de esconderijos para ratos e pessoas. Nesse sentido, o estudo de Pupo (1977) mostra aspectos relevantes sobre as pastagens e forrageiras incluindo de certa forma uma discussão sobre as plantas ruderais. Entretanto, deve-se salientar entre as espécies ruderais aquelas que têm também uma utilização popular como planta medicinal, fornecedora de produtos usados localmente, forrageiras, ornamentais, apícolas e até mesmo na alimentação humana.

Entre estas, pode-se salientar o melão-de-São-Caetano (Momordica charantia L.), quebra-pedra (Phyllanthus niruri L.), picão (Bidens pilosa L.) que têm uso na medicina popular e aquelas utilizadas ainda, como ornamentais, como a falsa-serralha (Emilia sonchifolia D.C.), margarida (Wedelia paludosa D.C.) e chanana (Turnera ulmifolia L.).

Entretanto, segundo Albuquerque (1993), as plantas invasoras, atualmente, são um grande problema para a agricultura por exigirem altos gastos com defensivos agrícolas poluentes. O homem, quando da conquista de novas terras, leva consigo seus implementos agrícolas, e junto a estes, frutos e/ou sementes de plantas consideradas indesejáveis.

Portanto, o objetivo deste estudo reside na catalogação das espécies ruderais que ocorrem em duas áreas urbanas da cidade de Cuiabá, MT, no sentido de ampliar o conhecimento das mesmas no Brasil.

\section{Material e métodos}

Dados gerais da cidade de Cuiabá e das áreas estudadas - O município de Cuiabá, MT, apresenta área de $8.122 \mathrm{Km}^{2}$, com altitude de 165 metros e coordenadas geográficas de $15^{\circ} 35^{\prime}$ e $56^{\prime \prime}$ de latitude S e 56 06' e $01^{\prime \prime}$ de longitude W de Greenwich (Diagnóstico florístico e faunístico da cidade de Cuiabá 1991). O clima da região é tropical, semiúmido, com duas estações definidas, seca (outono-inverno) e chuvosa (primaveraverão), do tipo $\mathrm{AW}$, na classificação de Köppen. As temperaturas elevadas definem médias em torno de $24^{\circ}$ a $26^{\circ} \mathrm{C}$. O solo é do tipo litólico (de pouca profundidade, aproximadamente $0.5 \mathrm{~m}$ ) e cambissolo (solo de pouco desenvolvimento pedológico). A vegetação autóctone é característica de cerrado, com áreas remanescentes principalmente na região periférica da cidade. As duas áreas estudadas, Campus da Universidade Federal de Mato Grosso e Bairro Boa Esperança, localizam-se no Coxipó da Ponte, ambas implantadas em regiões que configuravam basicamente o cerrado, atualmente, com fortes alterações. O Campus da Universidade Federal de Mato Grosso ainda conserva entre a sua vegetação, elementos florísticos que indicam espécies remanescentes do cerrado e da mata ciliar do córrego Barbado. Nessa área, vicejam espécies componentes da flora ruderal de cidades, como pode ser percebido através dos resultados deste estudo. O Bairro Boa Esperança, de classe média alta, apresenta inúmeros terrenos baldios, margem de ruas e algumas praças onde facilmente crescem as plantas ruderais.

Metodologia - Nas áreas selecionadas, as plantas foram coletadas em terrenos baldios, margens de ruas e avenidas, proximidades de residências e afins. Foram 
anotados, no campo, os dados referentes a floração, frutificação, nomes vulgares e usos regionais dos vegetais (estes, obtidos através de moradores e em consulta à literatura). Os vegetais foram processados no Laboratório de Botânica e Ecologia (Instituto de Biociências, Universidade Federal de Mato Grosso), onde realizou-se a triagem, a identificação e catalogação do material botânico coletado. As amostras testemunhas foram depositadas no Herbário da Universidade Federal de Mato Grosso (UFMT).

\section{Resultados e discussão}

A Tab. 1 mostra a relação de 109 espécies e 81 gêneros, distribuídos em 35 famílias, englobando as plantas ruderais coletadas e estudadas nas áreas selecionadas, bem como seus nomes vulgares e usos regionais, quando existentes na região

A família com maior número de espécies foi Gramineae (=Poaceae, com 14 espécies), logo após seguem Compositae (=Asteraceae, 13 espécies), Leguminosae (=Fabaceae, 11 espécies), Euphorbiaceae (10 espécies), resultados estes semelhantes aos obtidos por Gavilanes \& D’Angieri Filho (1991), na cidade de Lavras, MG e Mautone et. al. (1990), no Rio de Janeiro, que apresentam muitas das espécies catalogadas neste estudo, o que reforça a ampla ocorrência, distribuição e similaridade da flora ruderal em cidades brasileiras.

Entre as referências bibliográficas de ocorrência de plantas invasoras ou ruderais, em áreas urbanas, Kuhlmann et al. (1947) apresentam as espécies que ocorrem no Rio de Janeiro; Lisboa (1956) estudou as de Ouro Preto; Cervi \& Guimarães (1975) e Cervi et al. (1987, 1988) trataram daquelas da cidade de Curitiba; Pereira \& Agarez (1974, 1977) estudaram as ruderais da cidade do Rio de Janeiro.

Dentre as plantas ruderais estudadas, são encontradas várias espécies utilizadas pela população da cidade de Cuiabá. Estas espécies também foram encontradas por Gavilanes \& D’Angieri Filho (1991) na cidade de Lavras, em Minas Gerais. Dentre estas espécies, podem ser destacadas:

Momordica charantia L. (melão-de-São Caetano): as folhas desta planta clareiam as roupas e tiram nódoas. Os frutos novos são comestíveis, quer crus, em forma de saladas, ou cozidos, depois de separadas as sementes e escaldadas para tirar o gosto amargo. As folhas e frutos são vermífugos. A polpa das sementes misturada com vaselina, fornece ungüento usado para provocar a supuração em casos de tumores, furúnculos, etc. (Rodriguez s/d). Popularmente, os ramos e folhas são usados para preparar ninhos de galinhas poedeiras, com a finalidade de "espantar" piolhos.

Commelina nudiflora L. (trapoeraba-azul): é muito empregada na farmacopéia popular como diurética, antiblenorrágica e anti-reumática (Lorenzi 1991). Usada habitualmente pela população local como planta ornamental.

Ricinus communis L. (mamona): usualmente, o azeite, retirado das sementes, é usado para combater vermes intestinais (óleo de rícino). É uma das espécies de maior distribuição, ocorrendo tanto no Campus como no Bairro Boa Esperança.

Bidens pilosa L. (picão): suas principais propriedades medicinais abrangem: estimulante, antiescorbútica, antileucorréica, desobstruente do fígado, odontológica (raízes), vermífuga, antidisentérica (Lorenzi 1991). 
Tahela I - Relação das espécies ruderais catalogadas nas áreas estudadas na cidade de Cuiabá, seus nomes vulgares e usos regionais. PF- Planta Forrageira. PO- Planta Ornamental. PC- Planta Comestivel. PM- Planta Medicinal. FC- fruto comestivel. SO- sementes oleaginosas.

\begin{tabular}{|c|c|c|}
\hline Tixons & Nomes vulgares & Usos \\
\hline \multicolumn{3}{|l|}{ Acanthaceac } \\
\hline Ruellia squarrosa (Fenzl) Cufod & ruélia-roxal & $\mathrm{PO}$ \\
\hline \multicolumn{3}{|l|}{ Amaranthaceae } \\
\hline $\begin{array}{l}\text { Alternanthera ficoidea (L.) R. } \mathrm{Br} \\
\text { Amaranthus deflexus L. }\end{array}$ & apaga-fogo. perpétua -do mato & $\mathrm{PO}$ \\
\hline $\begin{array}{l}\text { Amaranthus deflexus } \mathrm{L} \text {. } \\
\text { A. spinosus } \mathrm{L} \text {. }\end{array}$ & caruru. caruru-rasteiro & PC \\
\hline $\begin{array}{l}\text { A. spinosus } \mathrm{L} \text {. } \\
\text { Gomphrena celosioides Mart. }\end{array}$ & caruru-de-porco & \\
\hline $\begin{array}{l}\text { Gomphrena celosioides Mart. } \\
\text { G. desertorum Mart. }\end{array}$ & perpétua. perpétua-do-mato & $\mathrm{PO}$ \\
\hline $\begin{array}{l}\text { G. desertorum Mart. } \\
\text { G. Ianigera Pohl ex Moq. }\end{array}$ & & \\
\hline $\begin{array}{l}\text { G. lanigera Pohl ex Moq. } \\
\text { A pocynaceae }\end{array}$ & & \\
\hline \multicolumn{3}{|l|}{ Apocynaceae } \\
\hline $\begin{array}{l}\text { Catharanthus roseus (L.) D. Don } \\
\text { Asclepiadaceae }\end{array}$ & maria-sem-vergonha, boa-noite & $\mathrm{PO}$ \\
\hline \multicolumn{3}{|l|}{ Asclepiadaceae } \\
\hline $\begin{array}{l}\text { Calotropis procera (Ait.) R. Br. } \\
\text { Bignoniaceae }\end{array}$ & algodão-de-seda. saco-de-vento & $\mathrm{PO}$ \\
\hline Bignoniaceae & & \\
\hline $\begin{array}{l}\text { Anemopaegma arvense (Vell.) Stelf. } \\
\text { Boraginaceac }\end{array}$ & verga-leso & $\mathrm{PM}$ \\
\hline \multicolumn{3}{|l|}{ Boraginaceac } \\
\hline Heliotropium indicum L. & garra-garra, borragem & \\
\hline H. transalpinum Vell. & borragem-hrava & \\
\hline Tournefortia paniculata Cham. & chá-mineiro & \\
\hline \multicolumn{3}{|l|}{ Capparidaceae } \\
\hline Cleome affinis DC. & mussambê & PO \\
\hline \multicolumn{3}{|l|}{ Cochlospermaceae } \\
\hline Cochlospermum regium (Mart \& Sch.) Pilger & algodão-do-campo & PO. PM \\
\hline \multicolumn{3}{|c|}{ Commelinaceac } \\
\hline Commelina benghalensis. L. & trapoeraba-azul & PO. PM \\
\hline C. nudiflora $\mathrm{L}$. & trapoeraba & PO, PM \\
\hline Commelina virginica $\mathrm{L}$. & trapoeraba & PO \\
\hline \multicolumn{3}{|l|}{ Compositac (=Asteraceac) } \\
\hline Acanthospermum australe (Loefl.) O. Kuntze & carrapicho-de-carneiro & PM \\
\hline A. hispidium DC. & espinho-de-carneiro & PM \\
\hline Ageratum conizoides $\mathrm{L}$. & mentrasto & $\mathrm{PM}$ \\
\hline Bidens pilosa $\mathrm{L}$. & picão & $\mathrm{PM}$ \\
\hline Emilia sonchifolia DC. & falsa-serralha & PO, PM \\
\hline Erigeron bonariensis $\mathrm{L}$. & erva-lancela & PM \\
\hline Mikania officinalis Mart. & chá-porreta & PM \\
\hline Orthopappus angustifolius (Sw.) Gleason & fumo-hravo & \\
\hline Porophyllum ruderale Cass. & picão-branco & PM \\
\hline Siegesbeckia orientalis L. & hotâo-de-ouro & $\mathrm{PO}$ \\
\hline Tridax procumbens $\mathrm{L}$. & beiço-de-hoi & \\
\hline Vernonia ferruginea Less. & assi-peixe & PM \\
\hline Wedelia paludosa DC. & margarida & PO \\
\hline \multicolumn{3}{|l|}{ Convolvulaceae } \\
\hline Ipomoea acuminata Roem. \& Sch. & campainha & $\mathrm{PO}$ \\
\hline 1. quamoclit $\mathrm{L}$. & cipó-esqueleto. esqueleto & PO. PM \\
\hline 1. purpurea Lam. & batata-brava. corda-de-viola & \\
\hline \multicolumn{3}{|l|}{ Cucurbitaceac } \\
\hline Cucumis anguria Roub. & maxixe & PM. FC \\
\hline Luffa aegyptica Mill. & bucha. esfregĩo & PM. FC \\
\hline Momordica charantia $\mathrm{L}$. & melão-de-Sã̌o Cactano & PM. FC \\
\hline
\end{tabular}


Tahela 1 - (continuação)

\begin{tabular}{|c|c|c|}
\hline Táxons & Nomes vulgares & Usos \\
\hline \multicolumn{3}{|l|}{ Cyperaceae } \\
\hline Cyperus brevifolius (Roub.) Hassk & tiririca & \\
\hline C. ferax L. C. Rich. & junquinho & PM \\
\hline C. flavus (Vahl) Ness. & liririca & \\
\hline C. luzulae (L.) Retz. & capim-hotão & $\mathrm{PF}$ \\
\hline Rhynchospora nervosa (Vahl) Boeck & capim-estrela & \\
\hline Scleria pterota Presi. & capim-navalha & \\
\hline \multicolumn{3}{|l|}{ Euphorbiaceae } \\
\hline Caperonia palustris(L.) St. Hil. & castanheiro-do brejo & \\
\hline Croton glandulosus(L.) M. Arg. & gervão-branco & \\
\hline C. lobatus $\mathrm{L}$. & café-bravo & \\
\hline Euphorbia heterophylla L & amendoim-bravo, leiterinho & \\
\hline E. hirta $\mathrm{L}$. & erva-de-andorinha,alcanjocira & PM \\
\hline E. hyssopifolia L. & erva-de-andorinha, azedinha & $\mathrm{PM}$ \\
\hline E. prostrata Ait. & & PM \\
\hline Manihot tripartita M. Arg. & mandioca-brava & \\
\hline Phyllanthus niruri $\mathrm{L}$. & quebra-pedra-rasteiro & PM \\
\hline Ricinus communis $\mathrm{L}$. & mamona & PM. SO \\
\hline \multicolumn{3}{|l|}{ Gramineae (=Poaceae) } \\
\hline Cenchrus echinatus L. & capim-carrapicho. timbete & \\
\hline Dactyloctenium aegyptium (L.) Beauv & capim-rastciro & PF \\
\hline Digitaria ciliaris (Retz) Koel. & capim-colchâo. milhã & \\
\hline D. horizontalis Willd & colchão-miúdo, capim-tinga & \\
\hline Eleusine indica (L.) Gaertn & capim-pé-de-galinha & PM. PF \\
\hline Hyparrhenia rufa (Ness) Stapf. & capim-jaraguá. provisório & \\
\hline Leptochloa filiformes ( Lam.) Beauv & capim-mimoso & \\
\hline Panicum maximun Jacq. & capim-colonião. capim-guiné & PF \\
\hline Paspalum conjugatum $\mathrm{L}$. & grama-de-forquilha. grama-doce & PF \\
\hline Rhynchelytrum repens (Willd.) Hubbart. & capim favorito & PF \\
\hline Setaria geniculata (Lam) Beauv. & capim-rabo-de-gato & PF \\
\hline S. poiretiana (Schult) Kunt & capim-amargoso & \\
\hline Sporobolus indicus (L.) R. Br. & capim-moirão. capeta & \\
\hline Trichachme insularis (L.) Ness. & & \\
\hline \multicolumn{3}{|l|}{ Labiatae (= Lamiaceac) } \\
\hline Hyptis brevipes Poit. & hortelã-brava. tapera-velha & PM \\
\hline \multicolumn{3}{|l|}{ Leguminosae $(=$ Fabaceae $)$} \\
\hline Arachis repens Handro & amendoim-de-jardim & PO \\
\hline Cassia occidentalis L. (=Senna) & fedegoso & PM \\
\hline C. tora L. (=Senna $)$ & mata-pasto-liso, fedegoso-bravo & OR \\
\hline \multicolumn{3}{|l|}{ Chamaecrista glandulosa (L.) Greene } \\
\hline Crotalaria anagyroides H. B. K & chocalho. guiso-de-cascavel & PF \\
\hline Crotalaria incana $\mathrm{L}$. & xique-xique, guiseiro & PF \\
\hline $\begin{array}{l}\text { Desmodium canum (Gmel.) Schinz \& Thel. } \\
\text { D. purpureum (Mill.) Fawc. et Rend. }\end{array}$ & $\begin{array}{l}\text { carrapicho-beiço-de-boi } \\
\text { carrapicho }\end{array}$ & PF. PM \\
\hline Mimosa pudica L & $\begin{array}{l}\text { carrapicho } \\
\text { dorme-dorme. malicia-de-mulher }\end{array}$ & PO \\
\hline Stylosanthes viscosa Sw. & meladinha. alfafa-nacional & PM \\
\hline Zornia latifolia $\mathrm{Sm}$. & urinária & \\
\hline \multicolumn{3}{|l|}{ Malvaceac } \\
\hline Hibiscus sabdariffa L. & quiabo-dc-angola & PM \\
\hline Pavonia spinefex (L.) Cav. & malva. arranca-estrepe & \\
\hline Sida cordifolia $\mathrm{L}$. & guanxuma. malva-branca & PM \\
\hline S. linifolia Cav. & malva-fina & \\
\hline
\end{tabular}


Tahela 1 - (continuação)

\begin{tabular}{|c|c|c|}
\hline Táxons & Nomes vulgares & Usos \\
\hline \multicolumn{3}{|l|}{ Malvaceae } \\
\hline S. rhombifolia L. & guanxuma. malva-preta & PM \\
\hline S. spinosa L. & malva-lanceta & PM \\
\hline \multicolumn{3}{|l|}{ Melastomataceae } \\
\hline Rhynchanthera leucorrhiza Moore & & $\mathrm{PO}$ \\
\hline \multicolumn{3}{|l|}{ Moraceae } \\
\hline $\begin{array}{l}\text { Cecropia peltata } \mathrm{L} \text {. } \\
\text { Nyctaginaceae }\end{array}$ & embaúva & PM \\
\hline Boerhavia diffusa $\mathrm{L}$. & pega-pinto. marra-pinto & PM \\
\hline \multicolumn{3}{|l|}{$\begin{array}{l}\text { Onagraceae } \\
\quad \text { Ludwigia sericeg (Camb) Hara }\end{array}$} \\
\hline Ludwigia sericea (Camb.) Hara & negreira-mirim, cruz-de-malta & $\mathrm{PO}$ \\
\hline $\begin{array}{l}\text { L. suffruticosa (L.) Hara } \\
\text { Oxalidaceae }\end{array}$ & negreira, cruz-de-malta & PO \\
\hline \multicolumn{3}{|l|}{$\begin{array}{l}\text { Oxalidaceae } \\
\text { Oxalis oxyptera Prog }\end{array}$} \\
\hline $\begin{array}{l}\text { Oxalis oxyptera Prog. } \\
\text { Piperaceae }\end{array}$ & azedinha & $\mathrm{PM}$ \\
\hline & jaborandi & PM \\
\hline $\begin{array}{l}\text { Polygalaceae } \\
\text { Polygala violacea Aubl. }\end{array}$ & & \\
\hline Portulacaceae & roxinha & PM \\
\hline Portulaca oleracea L. & beldroega. nove-horas & PO, PM \\
\hline Talinum patens (Jacq.) Willd. & lingua-de-vaca, erva-gorda & PC, PM \\
\hline \multicolumn{3}{|l|}{$\begin{array}{l}\text { Rubiaceae } \\
\text { Borreria suaveolens G F W }\end{array}$} \\
\hline Borreria suaveolens G. F. W. Meyer & poaia-do-cerrado & PM \\
\hline B. verticillata (L.) G. F. W. Meyer & vassourrinha-de-botão & $\mathrm{PO}$ \\
\hline $\begin{array}{l}\text { Manettia ignita Schum. } \\
\text { Scrophulariaceae }\end{array}$ & cipó-de-Santo Antônio & PM \\
\hline $\begin{array}{l}\text { Scoparia dulcis L. } \\
\text { Smilacaceae }\end{array}$ & vassourinha & PM \\
\hline $\begin{array}{l}\text { Smilax brasiliensis Spring } \\
\text { Solanaceae }\end{array}$ & japecanga & PM \\
\hline Physalis angulata $\mathrm{L}$. & juá-de-capote, poca, camapu & $\mathrm{FC}$ \\
\hline Solanum aculeatissimum Jacq. & joa-bravo, joá & PM \\
\hline $\begin{array}{l}\text { S. americanum Mill. } \\
\text { Sterculiaceae }\end{array}$ & maria-pretinha. erva-de-bicho & PM \\
\hline $\begin{array}{l}\text { Waltheria indica } \mathrm{L} \text {. } \\
\text { Turneraceae }\end{array}$ & malva-peluda & PM \\
\hline $\begin{array}{l}\text { Turnera ulmifolia } \mathrm{L} \text {. } \\
\text { yphaceae }\end{array}$ & chanana. Iurnera & $\mathrm{PO}$ \\
\hline $\begin{array}{l}\text { Thypha angustifolia L. } \\
\text { Jlmaceae }\end{array}$ & taboa. paina-de-flexa & PO, PM \\
\hline $\begin{array}{l}\text { Trema micrantha (L.) Blume } \\
\text { Ierbenaceae }\end{array}$ & periquiteira. crindiuva & PO, PM \\
\hline Lantana lilacina Desf. & cambará-roxo & $\mathrm{PO}$ \\
\hline Lippia alba (Mill) Brown & erva-cidreira-de-folha & PM \\
\hline
\end{tabular}

Sida rhombifolia L. (guanxuma): a infusão das folhas é empregada nas afecções das vias respiratórias. As folhas também se aplicam como emolientes e supurativas. Ludwigia suffruticosa (L.) Hara (cruz-de-malta): usada habitualmente em jardins, como planta ornamental. Prefere locais mais úmidos. 
Portulaca oleracea L. (beldroega): seu valor terapêutico é bem vasto tais como: mucilaginosa, diurética, laxativa, vermífuga e antiescorbútica (Lorenzi 1991). Em Cuiabá, os usos registrados foram como planta medicinal e ornamental.

Manettia ignita Schum. (cipó-de-Santo Antônio): em geral, ocorre em pastagens e sobre cercas. Nas áreas estudadas, principalmente no Bairro Boa Esperança, em terrenos baldios. A casca e as raízes são consideradas, pela população, eméticas e antidisentéricas.

Entre as espécies ruderais catalogadas, existem aquelas que têm uma ou outra utilização, como planta medicinal, forrageira, ornamental, comestível, sementes oleaginosas, situando-se nesse contexto, Cecropia peltata L., Trema micrantha (L.) Blume, Turnera ulmifolia L., Cochlospermum regium (Mart. \& Sch.) Pilger (espécie remanescente da vegetação de cerrado original), Cleome affinis DC. e Ipomoea quamoclit L.

\section{Referências bibliográficas}

Albuquerque, J. M. de. 1980. Identificação de plantas invasoras de cultura na região de Manaus. Acta Amazonica 10(1): 47-95.

Albuquerque, J. M. de. 1993. Identificação e germinação de sementes amazônicas. FCAP. Serviço de Documentação e Informação. Belém.

Amaral, J. K.; Carvalho, L. R. \& Caparelli, P. 1957. Principais invasoras de campos de pastagens e lavouras do Rio Grande do Sul. Boletim do Campo 97: 11-16.

Carvalho, L. F. 1958. Plantas invasoras de culturas no estado do Rio de Janeiro. Seminário Brasileiro de Herbicidas e Ervas Daninhas 2. Anais...p.115 a 124

Cervi, A. C. \& Guimarães, O. A. 1975 . Catálogo das plantas ruderais da cidade de Curitiba. Tribuna Farmacêutica 43(1-2): 1-20.

Cervi, A. C.; Guimarães, O. A.; Negrelle, R. R. B.; Sbalchiero, D. \& Conceição, M. C. A. 1987. Catálogo das plantas ruderais da cidade de Curitiba-PR. Estudos preliminares - II. Revista do Setor de Ciências Agrárias 9: 157-163.

Cervi. A. G.; Guimarães, O. A.; Acra, L. A.; Negrelle, R. R. B. \& Sbalchiero, D. 1988. Catálogo das plantas ruderais da cidade de Curitiba. Brasil. Estudos preliminares - III. Acta Biologica Paranaense 17(1,2,3,4): 109-139.

Correia, I. L. \& Chaves, M. J. 1972. Levantamento preliminar das plantas ruderais de João Pessoa. Anais do $23^{\circ}$ Congresso Nacional de Botânica. p. 327-329.

Diagnóstico florístico e faunístico da cidade de Cuiabá. 1991. Módulo II. Convênio FUFMT/ Prefeitura da Capital. Cuiabá. Relatório Final.

Ferreira. M. B. \& Laca-Buendia, J. P. de C. 1978. Espécies consideradas plantas daninhas em áreas cultivadas no Estado de Minas Gerais. Planta Daninha 1(2): 16-26.

Gavilanes, M. L. \& D’Angieri Filho, C. N. 1991. Flórula ruderal da cidade de Lavras, MG. Acta Botanica Brasilica 5(2):77-88.

Gonçalves, C. A.; Pimentel, D. M. \& Santos Filho, B. O. 1974. Plantas invasoras de pastagens do Estado do Pará. Boletim Técnico do IPEAN 62: 25-37.

Grime, J. P. 1982. Estratégias de adaptacion de las plantas y processos que controlam la vegetación. Ed. Limusa. México.

Kuhlmann, J. G.; Occhioni. P. \& Falcão, J. I. A. 1947. Contribuição ao estudo das plantas ruderais do Brasil. Arquivos do Jardim Botânico do Rio de Janeiro 7: 43-133,

Leitão Filho, H. F. de; Aranha, C. \& Bavhi, O. 1972. Plantas invasoras de culturas no Estado de São Paulo. vol 1. HUCITEC. São Paulo.

Lisboa, M. A. 1956. Aspectos da flora ruderal de Ouro Preto Pp. 133-146. Anais do V Congresso Nacional de Botânica. Sociedade Botânica do Brasil. Porto Alegre.

Lorenzi, H. 1984. Manual de identificação e controle de plantas daninhas. São Paulo. Piracicaba. Lorenzi, H. 1991. Plantas daninhas do Brasil: terrestres, aquáticas, parasitas, tóxicas e medicinais. $2^{\circ}$ Edição. Editora Plantarum. Nova Odessa. 
Mautone, L.; Brandão, M.; Guimarães, E. F. \& Miguel. J. R. 1990. Daninhas ocorrentes na zona serrana do Estado do Rio de Janeiro-Município de Petrópolis - 1. Acta Botanica Brasilica (2): 123-135.

Pereira, C. \& Agarez, F. V. 1974. Estudo das plantas ruderais do Estado da Guanabara. Leandra 3/4(45): $67-63$

Pereira, C. \& Agarez, F. V. 1977. Estudo das plantas ruderais do Estado do Rio de Janeiro - II. Leandra 6/7(7): 77-93

Pupo, N. I. H. 1977. Pastagens e forrageiras: pragas, doenças, plantas invasoras e tóxicas, controles. Instituto Campineiro de Ensino Agrícola. Campinas.

Rizzini, C. T. 1979. Tratado de Fitogeografia do Brasil - aspectos sociológicos e florísticos. $2^{\circ}$ Volume. HUCITEC/ EDUSP. São Paulo.

Rodriguez, R. H. s/d. Saúde através da natureza. A Terapêutica natural. Iª edição. Editora Miss. Luz para nossos Dias.

Snaydon, R. W. 1962. Microdistribution of Trifolium repens L. and its relation to soil factors. Journal of Ecology 50: 133-143. 